14

\title{
Нелинейная динамика паттернов импульсной активности ноцицептивных нейронов при коррекции повреждающего болевого воздействия
}

\author{
(C) O.E. Дик
}

Институт физиологии им. И.П. Павлова РАН, 199034 Санкт-Петербург, Россия

e-mail: dickviola@gmail.com

(Поступило в Редакцию 23 мая 2018 г.)

С помощью метода бифуркационного анализа модели ноцицептивного нейрона рассмотрена задача изменения структуры паттернов импульсной активности при возникновении антиноцицептивного ответа на повреждающее болевое воздействие в дорсальных ганглиях крысы. Обнаружено, что в модели может возникать эктопическая пачечная активность. Доказано, что подавление пачечной активности может быть связано исключительно с модификацией активационной воротной структуры медленных натриевых $N a_{V} 1.8$ каналов под действием анальгезирующего вещества, коменовой кислоты, являющейся лекарственной субстанцией нового неопиоидного анальгетика „аноцептина“.

DOI: 10.21883/JTF.2019.03.47187.200-18

\section{Введение}

Считается, что патофизиологической основой невропатической боли является гипервозбудимость ноцицептивных нейронов вследствие изменений в структурах, связанных с проведением и обработкой ноцицептивных сигналов [1-5]. При этом пароксизмальная боль, возникающая, например, при невралгии тройничного нерва, обусловлена эктопическими пачечными разрядами, генерируемыми поврежденными нервными волокнами [6]. Нормально низкий уровень электрической активности в нейронах дорсальных ганглиев, отмечаемый, как правило, в 75\% нейронов в виде медленных разрядов, многократно усиливается, когда эти разряды трансформируются в эктопические пачечные разряды при восприятии болевого повреждающего воздействия [7]. Основной вклад в генерацию импульсной активности, возникающей в ноцицептивных нейронах дорсальных ганглиев млекопитающих, вносят натриевые токи, задержанный калиевый $\left(I_{K}\right)$ ток и ток утечки $\left(I_{L}\right)$ [8]. Среди множества натриевых токов выделяют быстрый натриевый ток, быстро активирующийся и инактивирующийся тетродотоксин-чувствительный $\left(I_{N a f}\right)$; промежуточный натриевый ток, быстро активирующийся и медленнее инактивирующийся $\left(I_{N a I}\right)$; и медленный натриевый ток (еще медленнее инактивирующийся) $\left(I_{N a s}\right)$, генерируемые $N a_{V} 1.1, N a_{V} 1.7$ и $N a_{V} 1.8$ каналами соответственно [9]. Пачечная активность ноцицептивного нейрона может определяться динамикой двух токов, отличающихся наиболее медленной кинетикой $\left(I_{K}\right.$ и $\left.I_{N a s}\right)$. При этом ключевая роль в генерации болевой чувствительности отводится медленным натриевым каналам $N a_{V} 1.8$ [10], поскольку повышение синтеза этих каналов связано с повышением болевой чувствительности [11] и возникновением нейропатической боли [12]. В ряде работ установлено, что повреждение аксона нейронов спинальных ганглиев может приводить к снижению экспрессии мРНК калиевых каналов [13] и соответственно к снижению доли калиевых токов различной кинетики более чем на 60\% [14], а блокаторы калиевых каналов (тетраэтиламмоний и 4-аминопиридин) могут не подавлять, а, наоборот, усиливать ритмическую активность сенсорных нейронов [15-17]. Для того чтобы исключить влияние каналов задержанного калиевого тока на возникновение пачечной активности, мы в отличие от нашей предыдущей работы [18], в которой пачечная активность модели нейрона определялась динамикой двух медленных переменных, исключили влияние второй медленной переменной, связанной с кинетикой задержанного калиевого тока, полагая значение проводимости калиевых каналов $\left(g_{K}\right)$ равным нулю, что в эксперименте соответствовало бы блокированию этих каналов.

Цель настоящей работы - во-первых, определить механизмы изменения режимов импульсной активности ноцицептивных нейронов при коррекции повреждающего болевого воздействия и, во-вторых, доказать, что в условиях блокирования калиевого тока подавление пачечной активности может быть связано исключительно с модификацией активационной воротной структуры медленных натриевых $N a_{V} 1.8$ каналов под действием анальгезирующего вещества, коменовой кислоты, являющейся лекарственной субстанцией нового неопиоидного анальгетика „аноцептина“ [19].

\section{1. Модель ноцицептивного нейрона}

Модель мембраны ноцицептивного нейрона в условиях восприятия болевого сигнала может быть описана 

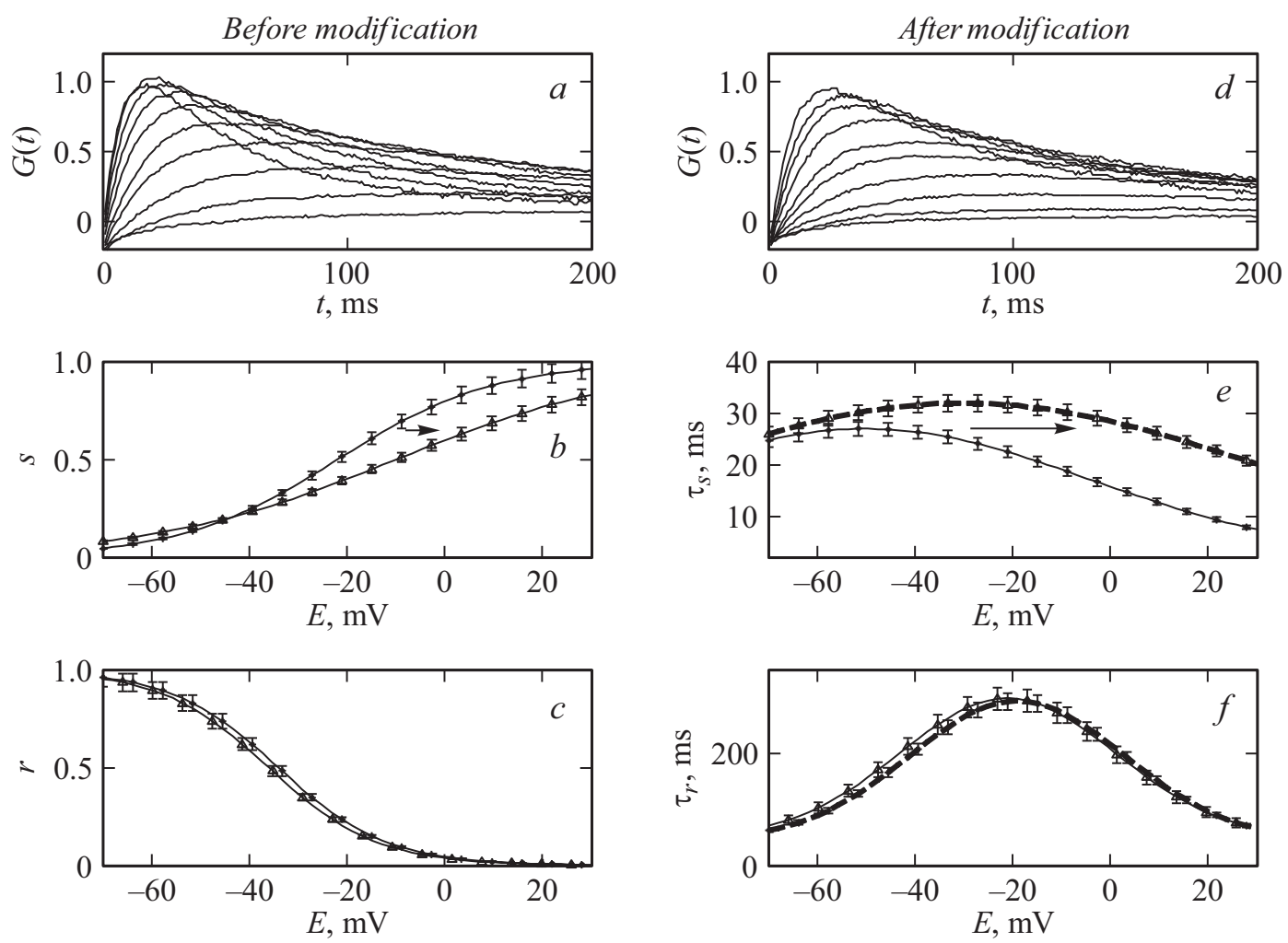

Рис. 1. Экспериментальные зависимости $G(t, E)$ и функции $s_{\infty}(E)$ и $\tau_{s}(E)$ для активации канала $N a_{V} 1.8$, а также зависимости $r_{\infty}(E)$ и $\tau_{r}(E)$ для его инактивации. Сплошные (штриховые) кривые соответствуют зависимостям до (после) модификации экспериментальных токов коменовой кислотой.

следующей системой уравнений:

$$
\begin{gathered}
\frac{d E}{d t}=\left(I-I_{N a f}(m, H, E)-E_{\text {nat }}(b, E)\right. \\
\left.-E_{L}(E)-I_{N a s}(s, t, E)\right) / c_{m}, \\
\frac{d x}{d t}=\left(x_{\infty}(E)-x\right) / \tau_{x}(E), \quad x=m, h, b, s, r,
\end{gathered}
$$

где $E$ - величина мембранного потенциала, $I-$ величина стимулирующего тока. Ионные токи определяются выражениями:

$$
\begin{gathered}
I_{N a f}=g_{N a} m^{3} h\left(E-E_{N a}\right), \\
E_{N a I}(b, E)=g_{N a I} m_{I \infty}(E) b\left(E-E_{N a}\right), \\
I_{N a S}(s, r, E)=g_{N a S} S^{3} r\left(E-E_{N a}\right),
\end{gathered}
$$

где переменные $m, h, b, s, r$ описывают воротные характеристики процессов активации и инактивации ионных каналов, константы $c_{m}=1 \mu \mathrm{F} / \mathrm{cm}^{2} ; g_{N a}=40 \mathrm{mS} / \mathrm{cm}^{2}$, $g_{N a I}=27 \mathrm{mS} / \mathrm{cm}^{2}, \quad g_{L}=1.4 \mathrm{mS} / \mathrm{cm}^{2}, \quad g_{N a S}=5 \mathrm{mS} / \mathrm{cm}^{2}$ определяют емкость мембраны, максимальные проводимости каналов быстрого и промежуточного натриевого токов, каналов утечки, а также каналов медленного натриевого тока; $E_{N a}=62 \mathrm{mV}, E_{L}=-77 \mathrm{mV}$ - равновесные потенциалы для $\mathrm{Na}^{+}$и ионов утечки. Потенциалзависимые стационарные и динамические характеристики воротных процессов всех каналов, кроме каналов
$N a_{V} 1.8$, детально описаны в работе [8]

$$
\begin{gathered}
m_{\infty}=1 . /(1 .+\exp (-(34.1+E) / 9.1)), \\
h_{\infty}=1 . /(q .+\exp ((56.4+E) / 72)), \\
m_{I \infty}=1 . /(1 .+\exp (-(25.3+E) / 9.1)), \\
b_{\infty}=1 . /(1 .+\exp ((72.5+E) / 8)), \\
\tau_{m}=0.01+0.11 \exp \left(-0.5\left(((E+28.7))^{2}\right)\right), \\
\tau_{h}=0.24+1.63 \exp \left(-\left(((E+61.9) / 15.3)^{2}\right)\right), \\
\tau_{m_{l}}=0, \\
\tau_{b}=0.22 \exp (-0.07 E) .
\end{gathered}
$$

Функции $s_{\infty}(E), r_{\infty}(E), \tau_{s}(E)$ и $\tau_{r}(E)$ для активации и инактивации медленных натриевых каналов $N a_{V} 1.8$ были определены по медленным натриевым токам, зарегистрированным в лаборатории физиологии возбудимых мембран Института физиологии им И.П. Павлова методом локальной фиксации потенциала в условиях плотного контакта в конфигурации целой клетки [20] на культивируемых изолированных нейронах спинальных ганглиев крыс. Исключение из вне- и внутриклеточного раствора ионов калия и наличие ионов фтора во внутриклеточном растворе обеспечивало устранение калиевых и кальциевых токов [21]. 
Экспериментальные зависимости $G(E, t)=I_{N a s}(E, t) /$ $\left(E-E_{N a}\right)$, полученные до и после воздействия на мембрану нейрона 5-гидрокси- $\gamma$-пирон-2-карбоновой (коменовой) кислоты, являющейся лекарственной субстанцией разрабатываемого нового неопиоидного анальгетика „аноцептина“ [2], в концентрации $100 \mathrm{nM} / 1$, представлены на рис. $1, a, d$.

Для нахождения параметров потенциало-зависимых функций $s_{\infty}(E), r_{\infty}(E), \tau_{s}(E), \tau_{r}(E)$, обеспечивающих наилучшее соответствие экспериментальным зависимостям $G(E, t)$, был использован многопараметрический метод наименьших квадратов. Минимизация функционала осуществлялась методом градиентного спуска. Сначала этим методом на основании формул

$$
\begin{gathered}
G(E, t)=g_{\max } s^{3}(E, t) r(E, t), \\
s(E, t)=s_{\infty}(E)\left(1-\exp \left(-t / \tau_{s}(E)\right)\right), \\
r(E, t)=r_{\infty}(E)+\left(1-r_{\infty}(E)\right) \exp \left(-t / \tau_{r}(E)\right),
\end{gathered}
$$

были найдены потенциало-зависимые функции $s_{\infty}(E)$, $r_{\infty}(E), \tau_{s}(E), \tau_{r}(E)$, характеризующие динамику процессов активации и инактивации медленного натриевого канала $N a_{V} 1.8$ до и после модификации экспериментальных токов коменовой кислотой (рис. $1, b, c, e, f$ ), далее определялись значения параметров, входящих в эти функции и обеспечивающих наилучшую аппроксимацию. Потенциалзависимые функции для активационной и инактивационной воротных структур $N a_{V} 1.8$ каналов до рассматриваемой модификации имели вид

$$
\begin{gathered}
s_{\infty}(E)=1 /(1+\exp (-(E+22) / 16)), \\
r_{\infty}(E)=1 /(1+\exp ((E+34) / 11)), \\
\tau_{s}(E)=2+25 \exp \left(-((E+50) / 65)^{2}\right), \\
\tau_{r}(E)=50+250 \exp \left(-((E+21) / 31)^{2}\right),
\end{gathered}
$$

а после модификации

$$
\begin{gathered}
s_{\infty}(E)=1 /(1+\exp (-(e+1-) / 25)), \\
r_{\infty}(E)=1 /(1+\exp ((e+32) / 11)), \\
\tau_{s}(E)=2+30 \exp \left(-((E+30) / 85)^{2}\right), \\
\tau_{r}(E)=45+250 \exp \left(-((E+19) / 31)^{2}\right) .
\end{gathered}
$$

Отметим, что изменения в инактивационной воротной структуре этих каналов после воздействия коменовой кислоты незначительны (рис. 1, $c, f$ ), и это вещество в основном влияло на активационную воротную систему медленного канала $N a_{V} 1.8$ (рис. $1, b, e$ ). На основании этих экспериментальных фактов в дальнейшем на модели были проанализированы результаты модификации только активационной воротной системы натриевого канала $N a_{V} 1.8$.

\section{2. Разложение системы на медленную и быструю подсистемы}

Для установления связей между типом устойчивого решения исследуемой системы (1), описывающей импульсную активность в ноцицептивных нейронах, значениями ее параметров и величиной внешнего стимула до и после анальгетической модификации активационной воротной системы канала $N a_{V} 1.8$, необходимо найти границу, разделяющую множество значений параметров модели на области качественно различных типов решений (стационарных состояний, в которых колебания отсутствуют или являются затухающими, и устойчивых периодических решений, при которых наблюдается периодическая импульсация) [22,23]. Для построения этой границы применялся метод бифуркационного анализа [24] и пакет программ MATCONT [25]. Численные решения системы (1) находились методом Рунге-Кутты четвертого порядка, модифицированным алгоритмом Гира с выбором переменного шага интегрирования. Примеры решений системы в виде периодических и пачечных колебаний с различной длительностью пачек и числом спайков в пределах одной пачки представлены на рис. 2.

Так как в режиме пачечной активности переходы между активной фазой, в которой наблюдаются пачечные разряды, и фазой их отсутствия, связаны с взаимодействием быстрых и медленных колебаний мембранного потенциала, для нахождения границы области пачечной активности, как правило, применяется разложение исходной системы на две подсистемы: медленную и быструю [26]. В рамках такого подхода возникновение быстрой компоненты пачечного разряда рассматривается как результат бифуркации быстрой подсистемы при изменении значений медленных переменных, которые выполняют роль бифуркационных параметров [27]. Эти параметры являются фазовыми координатами медленной подсистемы, а начало и конец пачечного разряда определяются прохождением траектории периодического решения быстрой подсистемы через бифуркационные значения параметров медленной подсистемы [27].

В связи с тем, что медленный натриевый ток имеет постоянную времени $\left(\tau_{r}\right)$, значительно превышающую постоянные времени для других токов (рис. 3), в качестве быстрой подсистемы будем рассматривать систему

$$
\begin{gathered}
b \frac{d E}{d t}=\left(I-I_{N a f}(m, h, E)-I_{N a I}(b, E)\right. \\
\left.-I_{L}(E)-I_{N a s}(s, r, E)\right) / c_{m}, \\
\frac{d x}{d t}=\left(x_{\infty}(E)-x\right) / \tau_{x}(E), \quad x=m, h, b, s,
\end{gathered}
$$

в которой переменная инактивации медленного натриевого тока $r$ является медленно меняющимся бифуркационным параметром. В таком случае медленная подсистема может быть записана в виде

$$
\begin{gathered}
I-I_{N a f}(m, h, E)-I_{N a I}(b, E)-I_{L}(E)-I_{N a s}(s, r, E)=0, \\
\frac{d r}{d t}=\left(r_{\infty}(E)-r\right) / \tau_{r}(E) .
\end{gathered}
$$



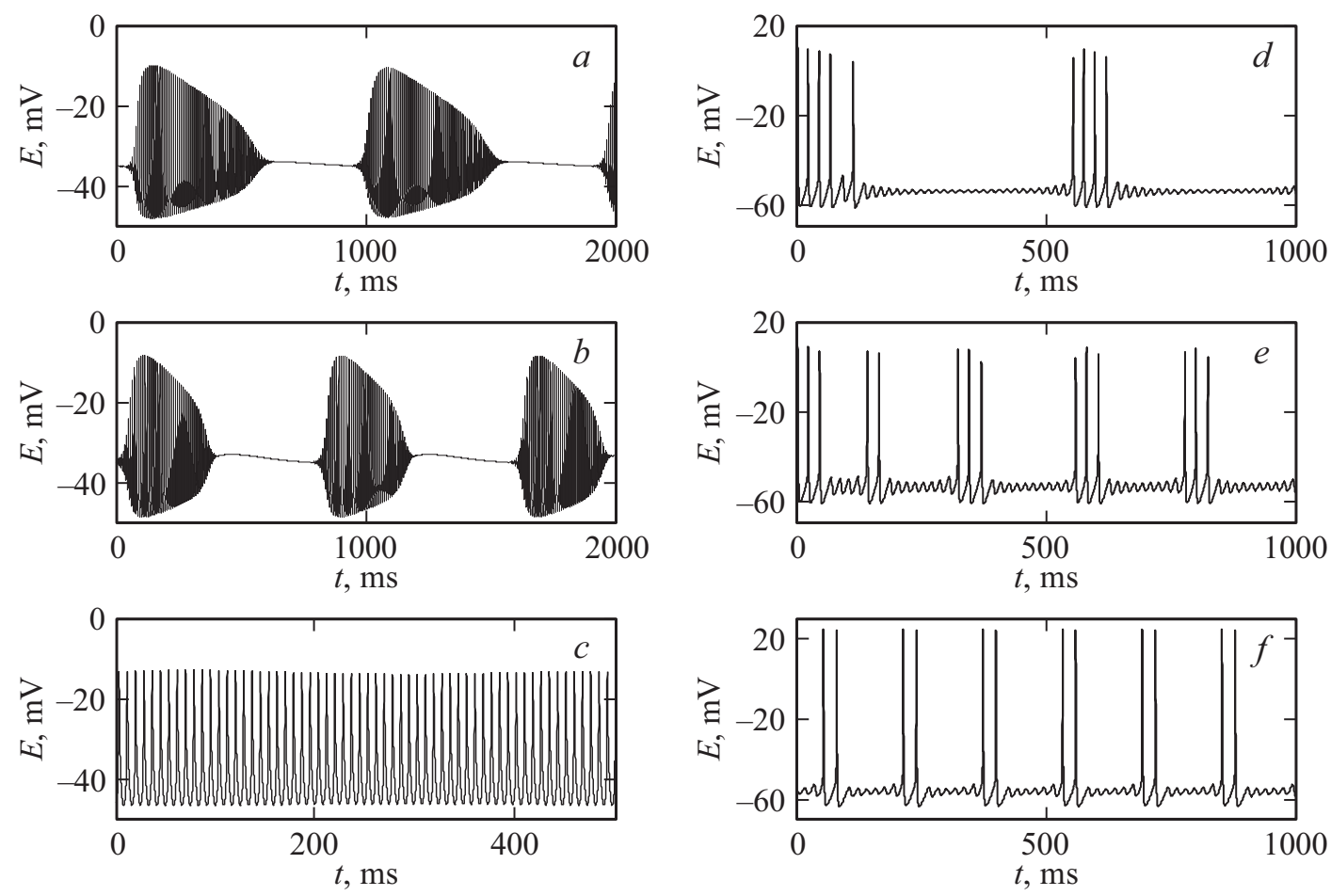

Рис. 2. Колебания модели для различных значений $g_{N a S}$ и $I$.
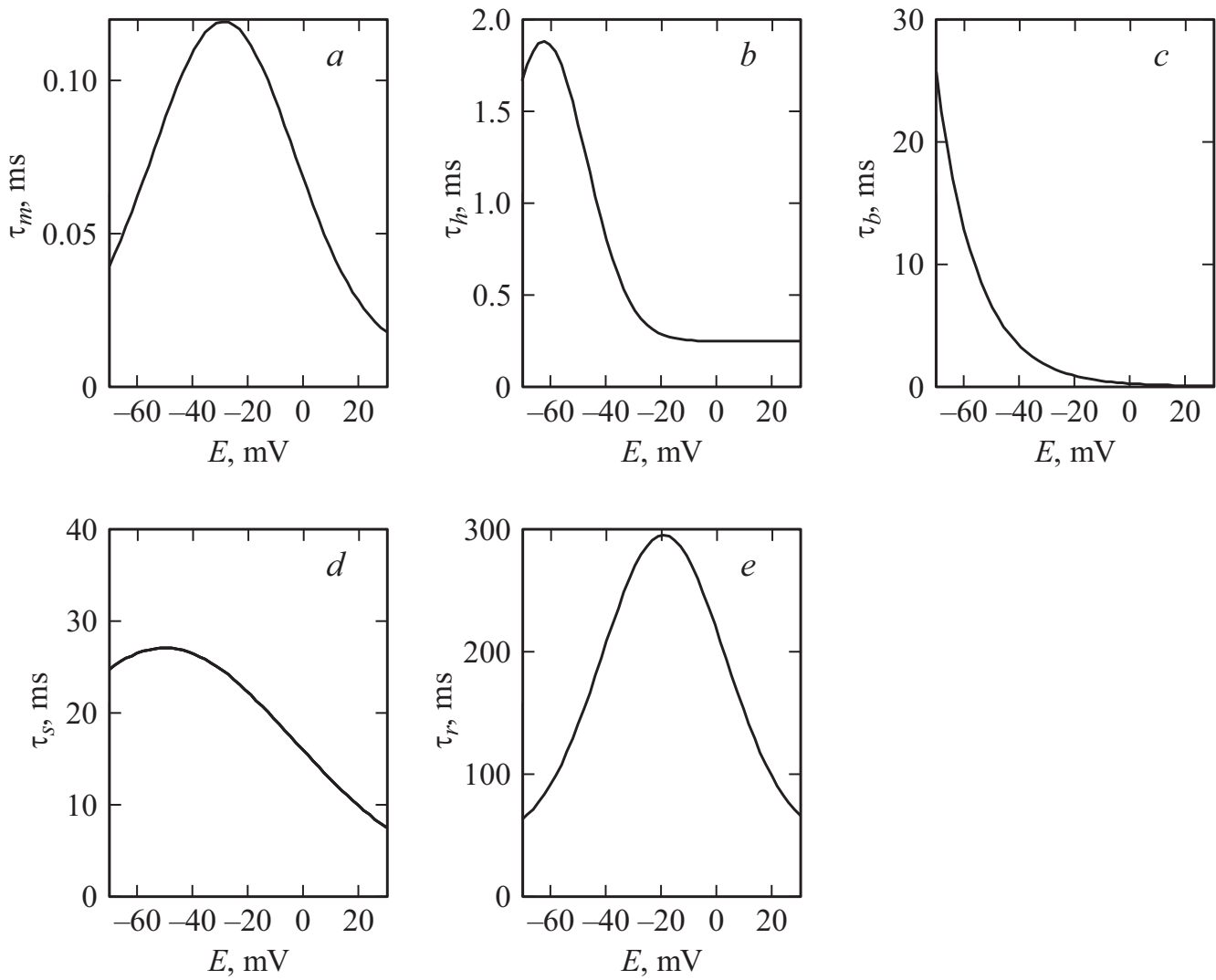

Рис. 3. Постоянные времени активации и инактивации быстрого, промежуточного и медленного натриевых токов. 

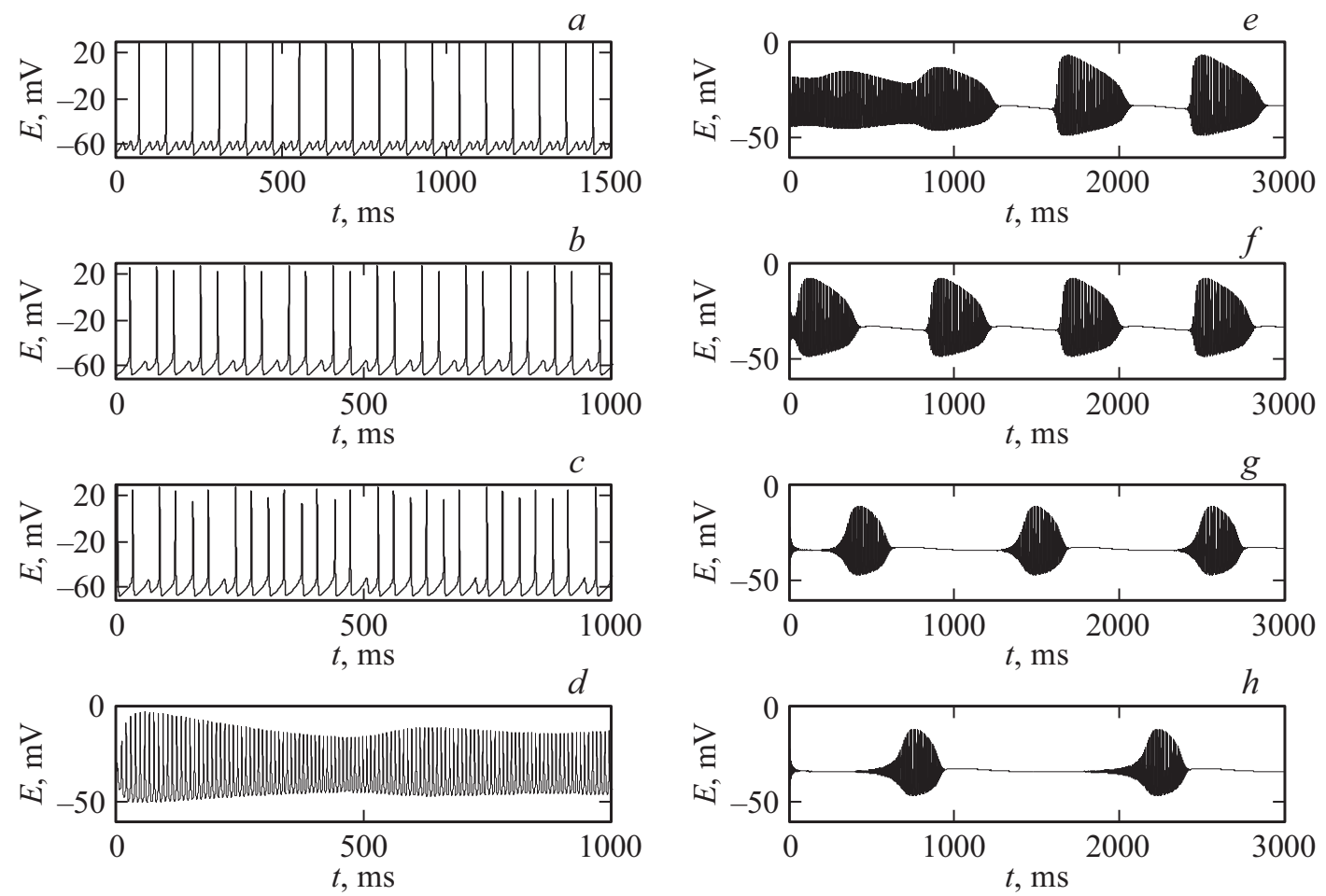

Рис. 4. Колебания модели при различных $I$ до модификации натриевых каналов $N a_{V} 1$.
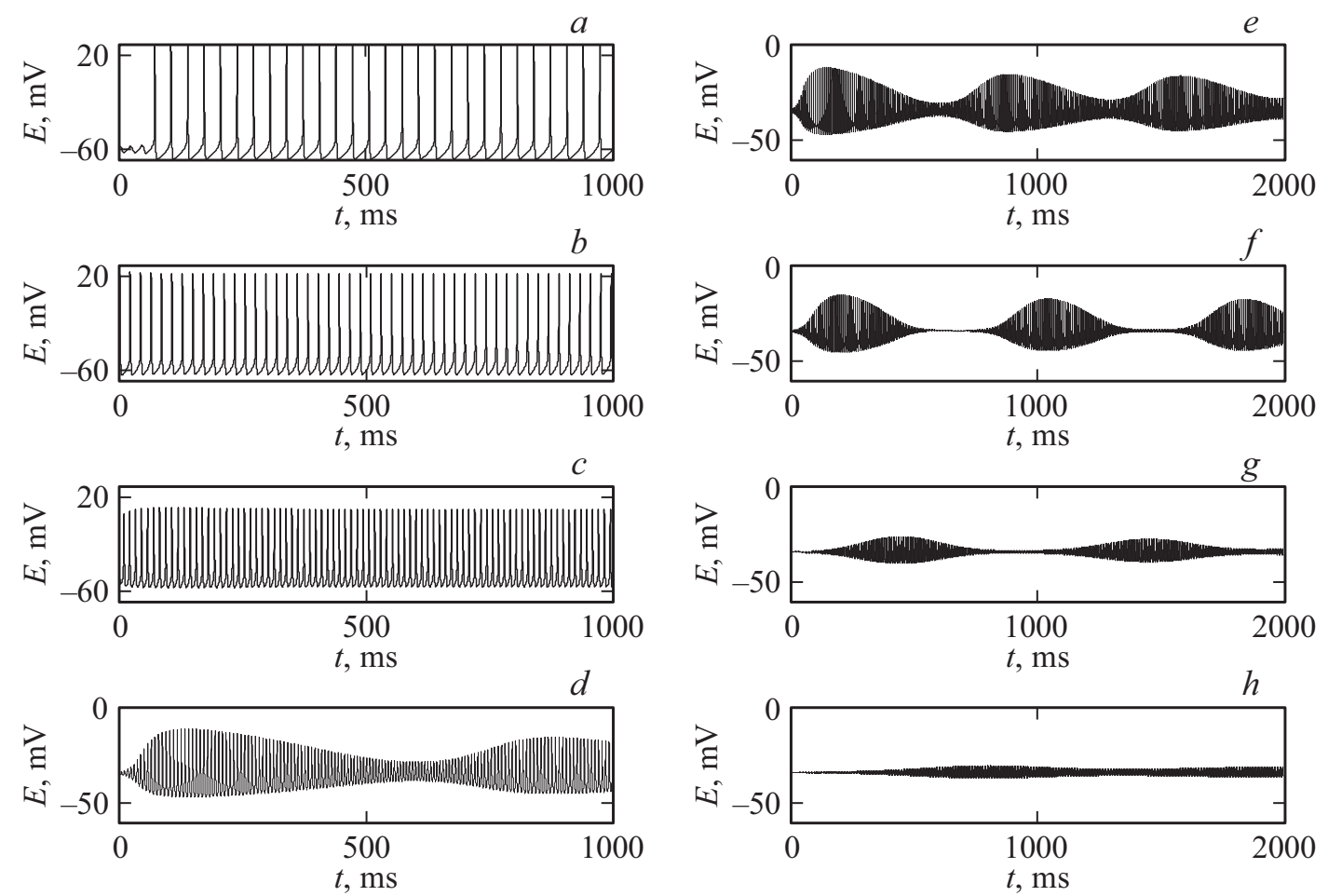

Рис. 5. Колебания модели при различных $I$ после модификации натриевых каналов $N a_{V} 1$.

При разложении исходной системы на медленную и быструю подсистемы начало активной фазы пачечного разряда соответствует потере устойчивости стационарного состояния быстрой подсистемы, а окончание актив- ной фазы соответствует потере устойчивости предельного цикла быстрой подсистемы [28]. Таким образом, для анализа изменений, происходящих в паттернах импульсной активности ноцицепторов в ответ на повреждающее 
болевое воздействие, необходимо исследовать соотношения между типом устойчивого решения исследуемой модели и величиной внешнего стимула до и после модификации модели путем построения однопараметрических бифуркационных диаграмм полной системы (1) на плоскости $(I, E)$. Для выяснения механизмов возникновения пачечной активности необходимо построение однопараметрических бифуркационных диаграмм быстрой подсистемы (2) на плоскости $(r, E)$.

\section{3. Результаты}

\section{1. Сравнение импульсной активности модели мембраны ноцицептивного нейрона до и после ее модификации}

Примеры колебаний модели мембраны ноцицептивного нейрона до модификации активационной структуры натриевых каналов $N a_{V} 1.8$ при различных значениях стимулирующего тока даны на рис. 4 . При $I=8 \mu \mathrm{A} / \mathrm{cm}^{2}$ модель демонстрирует синглетные колебания, в которых один импульс большой амплитуды чередуется с двумя подпороговыми колебаниями малой амплитуды (рис. 4, $a$ ). При $I=9.1 \mu \mathrm{A} / \mathrm{cm}^{2}$ наблюдаются колебания в виде дуплетов, в которых происходит чередование двух импульсов большой амплитуды с одним подпороговым колебанием (рис. 4,b). При увеличении стимулирующего тока возрастает число разрядов в пачке. Например, при $I=9.5 \mu \mathrm{A} / \mathrm{cm}^{2}$ наблюдается шесть импульсов в пачке, а число подпороговых колебаний не меняется при заданных значениях других параметров $\left(g_{N a}=40 \mathrm{mS} / \mathrm{cm}^{2}\right.$ и $\left.g_{N a S}=5.7 \mathrm{mS} / \mathrm{cm}^{2}\right)$ (рис. $\left.4, c\right)$. Затем режим пачечных колебаний сменяется на режим ритмических разрядов. Увеличение стимулирующего тока сопровождается возрастанием частоты этих разрядов. При $I=20.75 \mu \mathrm{A} / \mathrm{cm}^{2}$ периодические колебания становятся амплитудно-модулированными (рис. 4, $d$ ), а при $I=20.85 \mu \mathrm{A} / \mathrm{cm}^{2}$ наблюдается переход от амплитудномодулированных колебаний к пачечной активности с большим числом импульсов в пачке (рис. 4,e). Дальнейшее увеличение стимулирующего тока приводит к удлинению интервала между пачками, амплитуда и длительность пачек в этих колебаниях изменяется незначительно (рис. $4, f-h)$.

Примеры колебаний модели мембраны ноцицептивного нейрона после модификации активационной воротной структуры натриевых каналов $N a_{V} 1.8$ при различных значениях стимулирующего тока даны на рис. 5. При $I=7.54,13$ и $20 \mu \mathrm{A} / \mathrm{cm}^{2}$ модель демонстрирует быстрые колебания большой частоты и амплитуды (рис. 5, $a-c$ ). При $I=26.26$ и $26.3 \mu \mathrm{A} / \mathrm{cm}^{2}$ модель проявляет амплитудно-модулированные колебания, которые переходят в пачечные колебания при $I=26.5 \mu \mathrm{A} / \mathrm{cm}^{2}$ (рис. $5, d-f)$. При дальнейшем увеличении стимулирующего тока амплитуда пачек резко снижается (рис. 5,g $\left.I=26.51 \mu \mathrm{A} / \mathrm{cm}^{2}\right)$ и остаются низкоамплитудные высокочастотные модулированные колебания (рис. 5, $h$,
$\left.I=26.75 \mu \mathrm{A} / \mathrm{cm}^{2}\right)$. Таким образом, после модификации натриевых каналов $N a_{V} 1.8$ пачечная активность первого типа (в котором импульсы большой амплитуды чередуются с подпороговыми колебаниями) исчезает; наблюдаются переходы от быстрых периодических колебаний к амплитудно-модулированным колебаниям и от них к пачечной активности второго типа (с медленными колебаниями мембранного потенциала в интервалах между пачками и большим числом импульсов в пачке).

\section{2. Бифуркационный анализ смены режимов импульсной активности модели мембраны ноцицептивного нейрона при изменении величины стимула}

Для того чтобы определить значения стимулирующего тока, при которых происходят изменения режимов импульсной активности модели мембраны ноцицептивного нейрона, и сравнить эти изменения до и после модификации модели, построим бифуркационные диаграммы полной системы на плоскости $(I, E)$. На рис. 6 даны две такие бифуркационные диаграммы до (рис. 6, a) и после (рис. 6,b) модификации натриевых каналов $N a_{V} 1.8$. Бифуркационные диаграммы состоят из кривых стационарных состояний (тонкие кривые) и периодических орбит (толстые кривые). Устойчивые ветви стационарных состояний (сплошные линии) соответствуют гиперполяризованному состоянию покоя мембраны нейрона при значении мембранного потенциала $E \sim-61 \mathrm{mV}$ и деполяризованному состоянию покоя мембраны нейрона при значении мембранного потенциала $E \sim-34 \mathrm{mV}$.

Рассмотрим подробно бифуркационную диаграмму, изображенную на рис. $6, a$, при уменьшении величины стимулирующего тока. Потеря устойчивости стационарного состояния происходит в точке суперкритической бифуркации Андронова-Хопфа $\left(H_{1}\right)$ при значении стимула $I=23.49 \mu \mathrm{A} / \mathrm{cm}^{2}$, приводящей к возникновению устойчивого предельного цикла (периодической орбиты) малой амплитуды и большой частоты. По мере уменьшения стимула амплитуда устойчивых периодических орбит $\left(\left|E_{\max }-E_{\min }\right|\right)$ возрастает (рис. 6, $\left.a\right)$. При $I=23.483 \mu \mathrm{A} / \mathrm{cm}^{2}$ периодические орбиты теряют устойчивость в точке бифуркации Неймарка-Сакера (бифуркация тора) $\left(T R_{2}\right)$ и вместо периодических колебаний возникает сложное квазипериодическое движение на инвариантном торе. Колебания потенциала мембраны в этом случае являются амплитудо-модулированными. При $I=20.75 \mu \mathrm{A} / \mathrm{cm}^{2}$ существует вторая бифуркация Неймарка-Сакера $\left(T R_{1}\right)$. Таким образом, при значениях стимула внутри интервала $\left[I_{T R 1}, I T R_{2}\right]=[20.75$; $23.483] \mu \mathrm{A} / \mathrm{cm}^{2}$ наблюдаются пульсирующие паттерны с попеременно возрастающей и убывающей амплитудой импульсов. При $I<I_{T R 1}$ возникают устойчивые периодические колебания с более высокой амплитудой, но меньшей частотой, чем в интервале $\left[I_{T R 2}, H_{1}\right]$. При дальнейшем уменьшении величины стимулирующего тока определяются две бифуркации удвоения периода $\left(P D_{2}\right.$ 

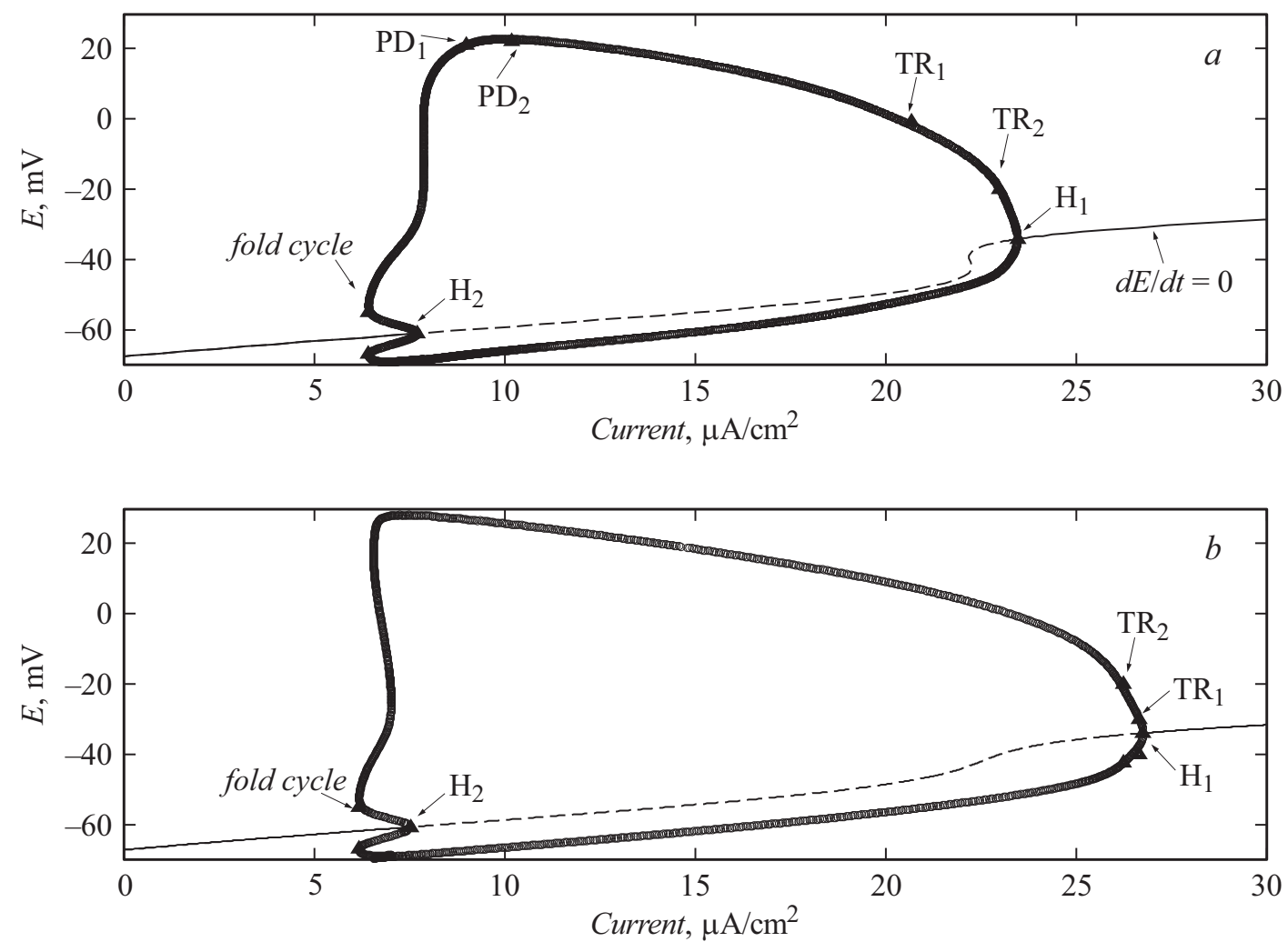

Рис. 6. Бифуркационные диаграммы полной системы на плоскости $(I, E)$ до $(a)$ и после $(b)$ модификации натриевых каналов $N a_{V} 1.8$.
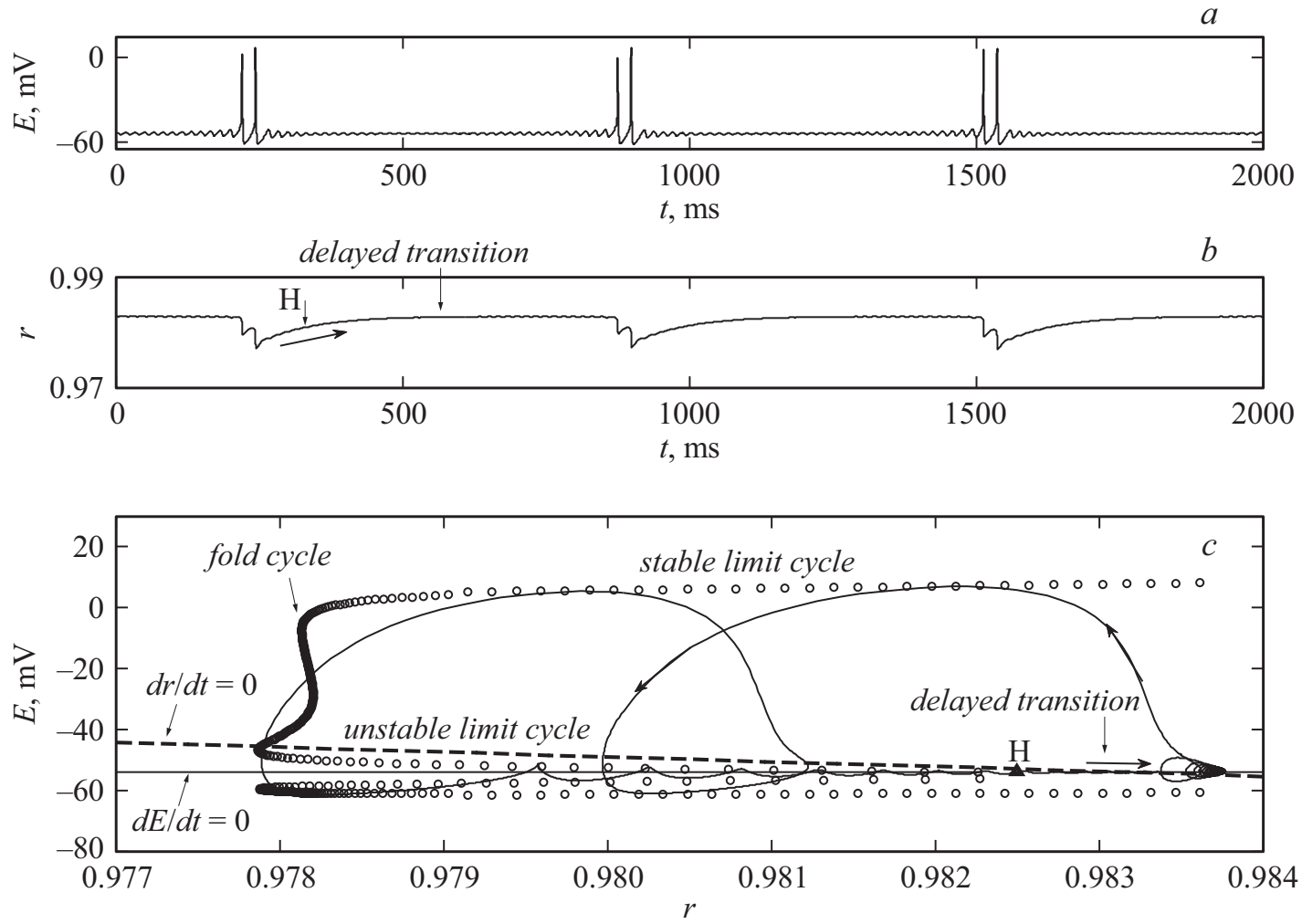

Рис. 7. Колебания быстрой $(E)$ и медленной $(r)$ переменных системы и бифуркационная диаграмма быстрой подсистемы на плоскости $(r, E)$ при значениях параметров $I=9.4 \mu \mathrm{A} / \mathrm{cm}^{2}, g_{N a S}=6.3 \mathrm{mS} / \mathrm{cm}^{2}$. 

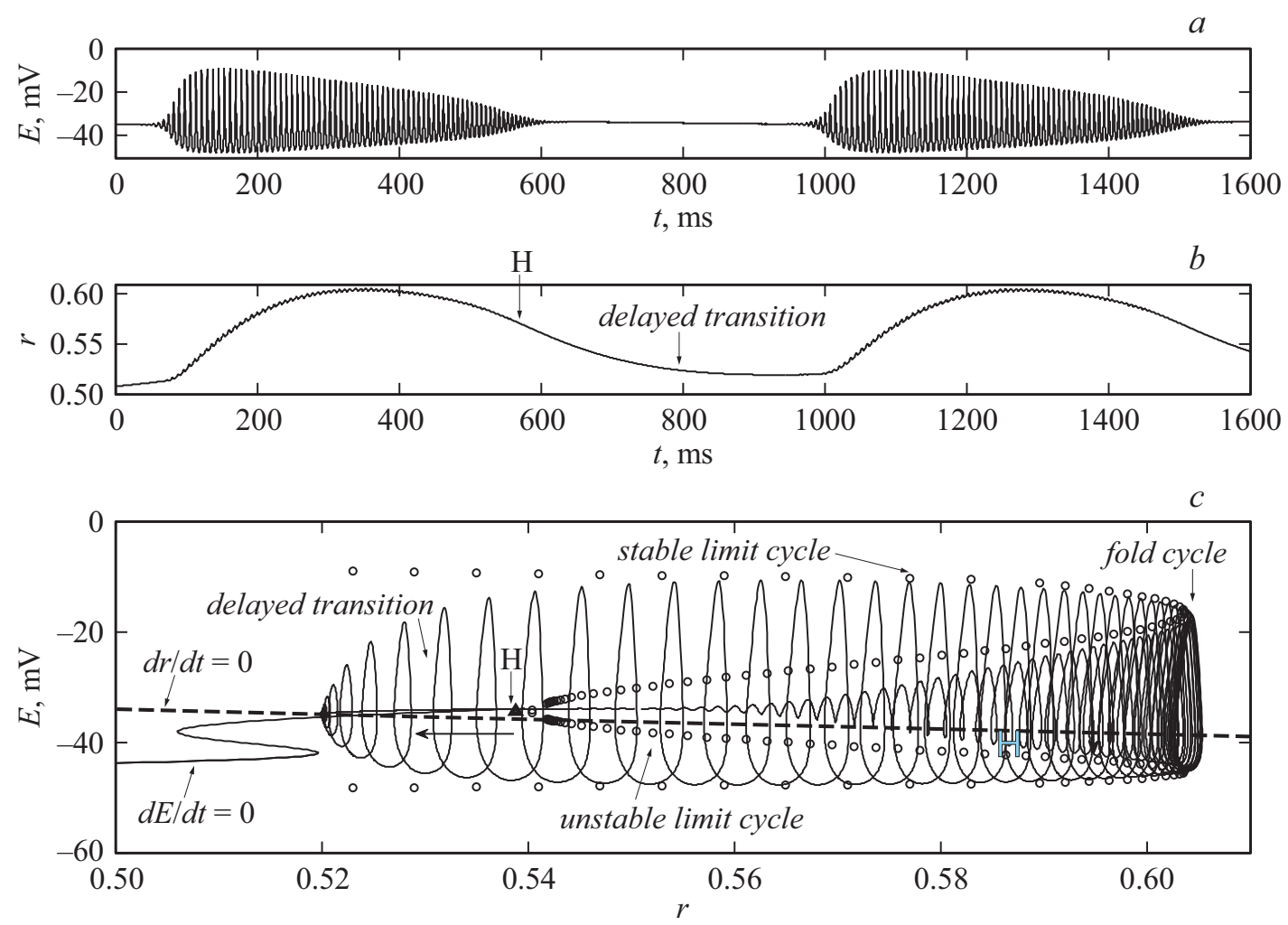

Рис. 8. Колебания быстрой $(E)$ и медленной $(r)$ переменных системы и бифуркационная диаграмма быстрой подсистемы на плоскости $(r, E)$ при значениях параметров $I=21 \mu \mathrm{A} / \mathrm{cm}^{2}, g_{\text {NaS }}=6.3 \mathrm{mS} / \mathrm{cm}^{2}$.

при $I=10.5 \mu \mathrm{A} / \mathrm{cm}^{2}$ и $P D_{1}$ при $\left.I=8.95 \mu \mathrm{A} / \mathrm{cm}^{2}\right)$. При $I=7.72 \mu \mathrm{A} / \mathrm{cm}^{2}$ наблюдается субкритическая бифуркация Андронова-Хопфа $\left(H_{2}\right)$, а вблизи нее находится седло-узловая бифуркация предельного цикла (LPC при $\left.I=6.59 \mu \mathrm{A} / \mathrm{cm}^{2}\right)$, в силу чего в интервале значений стимула $\left[I_{L P C}, I_{H 2}\right]$ сосуществуют устойчивый и неустойчивый предельные циклы, а в седло-узловой бифуркации предельного цикла эти периодические орбиты сливаются и исчезают, уступая место стационарному состоянию.

После модификации натриевых каналов $N a_{V} 1.8$ бифуркационная диаграмма полной системы не содержит точек удвоения периода, а интервал значений $\left[I_{T R 2}, I_{T R 1}\right]=[26.26 ; 26.52] \mu \mathrm{A} / \mathrm{cm}^{2}$ между двумя бифуркациями тора резко сужается, поэтому большую часть области бифуркационной диаграммы занимают быстрые периодические колебания (рис. $6, b$ ). Таким образом, показано, что после модификации активационной воротной структуры медленных натриевых каналов пачечные разряды с подпороговыми осцилляциями в интервалах между пачками исчезают, а диапазон значений стимула, в котором существуют быстрые ритмические колебания, значительно увеличивается. Следовательно, в условиях блокирования калиевого тока после модификации коменовой кислотой медленных натриевых каналов $N a_{V} 1.8$ пачечная активность первого типа подавляется, а пачечная активность второго типа сохраняется, но диапазон значений стимула, при которых она возможна, значительно уменьшается.

\section{3. Механизм возникновения пачечной активности в модели мембраны ноцицептивного нейрона}

Рассмотрим механизм возникновения пачечной активности первого типа в модели нейрона дорсального ганглия. Для этого построим однопараметрическую бифуркационную диаграмму быстрой подсистемы на плоскости $(r, E)$ при значениях параметров $I=9.4 \mu \mathrm{A} / \mathrm{cm}^{2}$, $g_{\text {NaS }}=6.3 \mathrm{mS} / \mathrm{cm}^{2}$ (рис. 7, $c$ ), на которой величина $r$ является бифуркационным параметром. Бифуркационная диаграмма включает в себя ветви стационарных состояний (кривые, отмеченные, $d E / d t=0^{\text {“ }}$ и,$d r / d t=0^{\circ “}$ ) и периодических орбит. Неустойчивые периодические орбиты обозначены „,unstable limit cycle“, устойчивые орбиты - „stable limit cycle“. На бифуркационную диаграмму наложена траектория полной системы.

Колебания быстрой $(E)$ и медленной $(r)$ переменных системы, соответствующие построенной бифуркационной диаграмме, представлены на рис. 7, $a, b$. Во время пачечного разряда значение переменной $r$ падает, в то время как в периоды между пачками переменная $r$ медленно нарастает. В интервале между пачками траектория полной системы движется в сторону увеличения параметра $r$ вдоль ветви стационарных состояний быстрой подсистемы (рис. 7,c). Активная фаза пачечной активности начинается, когда эта траектория проходит через точку субкритической бифуркации Андронова-Хопфа быстрой подсистемы (обозначенную Н). В этой точке 
стационарное состояние полной системы теряет устойчивость, и система с некоторой задержкой переходит в режим устойчивых периодических колебаний большой амплитуды. Затем траектория движется влево, пока не достигнет седло-узловой бифуркации предельного цикла ( обозначенной „fold cycle“). Наконец, траектория полной системы возвращается к $E$-нульклине $(d E / d t=0)$ и затухающим колебаниям малой амплитуды, а от них снова через бифуркацию Андронова-Хопфа к колебаниям большой амплитуды. Рассмотренная динамика позволяет утверждать, что исследуемый тип пачечной активности представляет собой тип пачечного разряда „Хопф/складка“ („subHopf /fold cycle burster“) по топологической классификации моделей пачечной активности Ижикевича [29], так как активная фаза пачечной активности начинается в точке субкритической бифуркации Андронова-Хопфа и заканчивается в седло-узловой бифуркации предельного цикла быстрой подсистемы. Отметим, что именно такой тип пачечной активности, как правило, развивается в поврежденных сенсорных нейронах после травмы и инициируется подпороговыми колебаниями мембранного потенциала [19,30].

Рассмотрим механизм возникновения пачечной активности второго типа, построив бифуркационную диаграмму быстрой подсистемы на плоскости $(r, E)$ при значениях параметров $I=21 \mu \mathrm{A} / \mathrm{cm}^{2}, g_{\mathrm{NaS}}=6.3 \mathrm{mS} / \mathrm{cm}^{2}$ (рис. 8,c). Колебания быстрой $(E)$ и медленной $(r)$ переменных системы, соответствующие построенной бифуркационной диаграмме, представлены на рис. 8, $a, b$. Во время пачечного разряда значение переменной $r$ медленно возрастает, а в периоды между пачками падает, однако эта бифуркационная диаграмма имеют такую же структуру типа „Хопф/складка“ [29], как и диаграмма, представленная на рис. 7,c. Активная фаза пачечной активности начинается при прохождении траектории полной системы через точку субкритической бифуркации Андронова-Хопфа быстрой подсистемы. В этой точке система скачком переходит в режим колебаний с нарастающей амплитудой и после некоторой задержки (на рис. 8,c это движение влево) достигает ветви устойчивых периодических колебаний большой амплитуды. Затем траектория полной системы движется до седло-узловой бифуркации предельного цикла (на рис. 8, с это движение вправо), после чего активная фаза пачечной активности заканчивается.

\section{4. Выводы}

В основе изменения паттернов импульсной активности ноцицепторов при коррекции повреждающего болевого воздействия лежат перестройки во временной организации паттернов за счет бифуркаций стационарных состояний и предельных циклов, приводящих к появлению режима пачечной активности. Механизм возникновения пачечной активности в условиях блокирования калиевого тока связан с прохождением траектории системы через бифуркацию Андронова-Хопфа и седлоузловую бифуркацию предельного цикла. Этот механизм характерен как для первого, так и для второго типа наблюдаемой пачечной активности. В основе механизма коррекции повреждающего болевого воздействия лежит молекулярный механизм подавления пачечных разрядов, связанный с модификацией активационной воротной структуры медленных натриевых $N a_{V} 1.8$ каналов под действием анальгезирующего вещества, при этом важнейшая физиологическая функция генерации импульсной активности не блокируется полностью.

Работа выполнена при финансовой поддержке Программы фундаментальных научных исследований государственных академий на 2013-2020 годы (ГП-14, раздел 64).

\section{Список литературы}

[1] Devor M., Seltzer Z. (In: Wall P.D., Melzack R., eds. Textbook of pain. 4.) // London: Churchill Livingstone, 1999. C. $129-164$.

[2] Liu C.N., Michaelis M., Amir R. et al. // J. Neurophysiol. 2000. Vol. 84. P. 205-215.

[3] Liu C.N., Wall P.D., Ben-Dor E. et al. // Pain. 2000. Vol. 85. P. 503-521.

[4] Zhang J.M., Li H., Brull S.J. // J. Neurophysiol. 2000. Vol. 84. P. $798-80$.

[5] Boucher T.J., McMahon S.B. // Curr Opin Pharmacol. 2001. P. 66-72.

[6] Devor M. // Exp. Brain Res. 2009. Vol. 196. P. 115-128.

[7] Amir R., Michaelis M., Devor M. // J. Neurosci. 2002. Vol. 22. P. 1187-1198.

[8] Kovalsky Y., Amir R., Devor M. // J. Neurophysiol. 2009. Vol. 102. P. $1430-1442$.

[9] Elliott A.A., Elliott J.R. // J. Physiol. 1993. Vol. 463. P. 39-56.

[10] Kostyuk E.P., Kostyuk P.G., Voitenko N.V. // Neurophysiol. 2001. Vol. 33. P. 303-313.

[11] Goldin E. // J. Exper. Biol . 2000. Vol. 205. P. 575-584.

[12] Waxman S.G., Cummins T.R., Dib-Haij S.D. et al.// J. Rehabil Res. Dev. 2000. Vol. 37. P. 517-528.

[13] Ishikawa K., Tanaka M., Black J. et al. // Muscle Nerve. 1999. Vol. 22. P. 502-507.

[14] Everill B., Kocsis J.D. // J. Neurophysiol. 1999. Vol. 82. P. 700-708.

[15] Devor M. // Neurosci Lett. 1983. Vol. 40. P. 181-186.

[16] Kocsis J.D., Bowe C.M., Waxman S.G. // Neurology. 1986. Vol. 36. P. $117-120$.

[17] Amir R., Liu C.N., Kocsis J.D. et al. // Brain. 2002. Vol. 125. P. 421-435.

[18] Дик О. Е. // ЖТФ. 2017. Т. 87. Вып. 11. С. 1742-1747.

[19] Krylov B.V., Rogachevskii I.V., Shelykh T.N. et al. // Sharjah: Bentham sci. publ. 2017. 203 C. (Frontiers in pain sci. Vol. 1.)

[20] Hamill O.P., Marty A., Neher E. // Pflug. Arch. 1981. Vol. 391. P. $85-100$.

[21] Kostyuk P.G., Veselovsky N.S., Tsyndrenko A.Y. // Neuroscience. 1981. Vol. 6. P. 2423-2430.

[22] Bedrov Y.A., Akoev G.N., Dick O.E. // Biolog. Cybern. 1992. Vol. 66. P. 413-418.

[23] Fukai H., Doi S., Nomura T. et al. // Biolog. Cybern. 2000. Vol. 82. P. 215-222. 
[24] Kuznetsov Y.A. Elements of Applied Bifurcation Theory. NY: Springer Verlag, 1995.

[25] Dhooge A., Govaerts W., Kuznetsov Y.A. et al. MatCont and CL_Matcont Continuation toolboxes in MATLAB, Utrecht Univ, Netherlands, 2006.

[26] Guckenheimer J., Harris-Warrick R.M., Peck J. et al. // J. Comp. Neurosci. 1997. Vol. 4. P. 257-277.

[27] Rinzel J., Lee Y.S. In: Nonlinear Oscillations in Biology and Chemistry. Ed. by Othmer H.G. Lecture Notes in Biomathematics. NY:: Springer-Verlag, 1986.

[28] Guckenheimer J., Holmes D. Nonlinear Oscillations, Dynamical Systems, and Bifurcations of Vector Fields. NY: Springer-Verlag, 1983.

[29] Izhikevich E.M. // J. Bifur Chaos. 2000. Vol. 10. P. $1171-1266$.

[30] Kovalsky Y., Amir R., Devor M. // Exp. Neurol. 2008. Vol. 210. P. $194-206$ 